\title{
Metabolomic profiling identifies novel biomarkers and mechanisms in human bladder cancer treated with submucosal injection of gemcitabine
}

\author{
CHAO YANG $^{1 *}$, XIAN SUN $^{2,3^{*}}$, HENGBING WANG $^{1 *}$, TING LU $^{2,3}$, KEQING WU ${ }^{2,3}$, \\ YUSHENG GUAN $^{2,3}$, JING TANG ${ }^{1}$, JIAN LIANG ${ }^{4}$, RONGLI SUN ${ }^{5}$, ZHONGYING GUO $^{6}$, \\ SINIAN ZHENG ${ }^{7}$, XIAOLI WU ${ }^{8}$, HESONG JIANG $^{1}$, XI JIANG $^{1}$, BING ZHONG ${ }^{1}$, XIAOBING NIU ${ }^{1}$, \\ SUAN SUN ${ }^{6}$, XINRU WANG ${ }^{2,3}$, MINJIAN $\mathrm{CHEN}^{2,3}$ and GUANGBO FU ${ }^{1}$ \\ ${ }^{1}$ Department of Urology, The Affiliated Huai'an No. 1 People's Hospital of Nanjing Medical University, \\ Huai'an, Jiangsu 223300; ${ }^{2}$ State Key Laboratory of Reproductive Medicine, Center for Global Health;
}

${ }^{3}$ Key Laboratory of Modern Toxicology of Ministry of Education, School of Public Health, Nanjing Medical University, Nanjing, Jiangsu 211166; ${ }^{4}$ Center of Reproduction and Genetic, The Affiliated Huai'an No. 1 People's Hospital of Nanjing Medical University, Huai'an, Jiangsu 223300; ${ }^{5}$ Key Laboratory of Environmental Medicine Engineering, Ministry of Education, School of Public Health, Southeast University, Nanjing, Jiangsu 210009; ${ }^{6}$ Department of Pathology, The Affiliated Huai'an No. 1 People's Hospital of Nanjing Medical University, Huai'an, Jiangsu 223300; ${ }^{7}$ Department of Urology, Ningbo Medical Center Lihuili Eastern Hospital, Ningbo, Zhejiang 315040; ${ }^{8}$ Department of Pharmacy, The Affiliated Huai'an No. 1 People's Hospital of Nanjing Medical University, Huai'an, Jiangsu 223300, P.R. China

Received April 18,2019; Accepted September 6, 2019

DOI: $10.3892 /$ ijmm.2019.4347

\begin{abstract}
Bladder cancer (BCa) is a common urinary tract malignancy with frequent recurrences after initial resection. Submucosal injection of gemcitabine prior to transurethral
\end{abstract}

Correspondence to: Professor Guangbo Fu, Department of Urology, The Affiliated Huai'an No. 1 People's Hospital of Nanjing Medical University, 1 West Huanghe Road, Huai'an, Jiangsu 223300, P.R. China

E-mail: fgb200@vip.163.com

Professor Minjian Chen, State Key Laboratory of Reproductive Medicine, Center for Global Health, School of Public Health, Nanjing Medical University, 818 East Tianyuan Road, Nanjing, Jiangsu 211166, P.R. China

E-mail: minjianchen@njmu.edu.cn

*Contributed equally

Abbreviations: TURBT, transurethral resection of bladder tumor; PCA, principal component analysis; ACN, acetonitrile; KEGG, Kyoto Encyclopedia of Genes and Genomes; HMDB, human metabolome database; SMPDB, Small Molecule Pathway Database; HPLC/MS, high-performance liquid chromatography/ mass spectrometry; UPLC-MS, ultra-performance LC-MS; UPLC-TOF-MS, UPLC time-of-flight MS; UPLC-HRMS, UPLC high-resolution MS; BCa, bladder cancer

Key words: tissue metabolomics, submucosal injection, gemcitabine, biomarkers, bladder cancer resection of bladder tumor (TURBT) may prevent recurrence of urothelial cancer. However, the underlying mechanism remains unknown. In the present study, ultra-performance liquid chromatography Q-Exactive mass spectrometry was used to profile tissue metabolites from $12 \mathrm{BCa}$ patients. The 48 samples included pre- and post-gemcitabine treatment $\mathrm{BCa}$ tissues, as well as adjacent normal tissues. Principal component analysis (PCA) revealed that the metabolic profiles of pre-gemcitabine BCa tissues differed significantly from those of pre-gemcitabine normal tissues. A total of 34 significantly altered metabolites were further analyzed. Pathway analysis using MetaboAnalyst identified three metabolic pathways closely associated with $\mathrm{BCa}$, including glutathione, purine and thiamine metabolism, while glutathione metabolism was also identified by the enrichment analysis using MetaboAnalyst. In search of the possible targets of gemcitabine, metabolite profiles were compared between the pre-gemcitabine normal and post-gemcitabine $\mathrm{BCa}$ tissues. Among the 34 metabolites associated with $\mathrm{BCa}$, the levels of bilirubin and retinal recovered in $\mathrm{BCa}$ tissues treated with gemcitabine. When comparing normal bladder tissues with and without gemcitabine treatment, among the 34 metabolites associated with $\mathrm{BCa}$, it was observed that histamine change may be associated with the prevention of relapse, whereas thiamine change may be involved in possible side effects. Therefore, by employing a hypothesis-free tissue-based metabolomics study, the present study investigated the metabolic signatures of $\mathrm{BCa}$ and found that bilirubin and retinal may be involved in the mechanism underlying the biomolecular action of submucosal injection of gemcitabine in urothelial BCa. 


\section{Introduction}

Bladder cancer $(\mathrm{BCa})$ ranks ninth among the most common solid tumors worldwide (1). Approximately $75 \%$ of newly diagnosed $\mathrm{BCa}$ cases are non-muscle invasive, and the majority are histologically low-grade cancer (2). Routine surveillance to monitor $\mathrm{BCa}$ recurrence includes cystoscopic examination due to a high risk of recurrence after initial resection. The repeat transurethral resection of bladder tumor (TURBT) remains the first-line treatment for BCa recurrence (3). However, all these invasive procedures result in a high cost of care, and are often associated with significant morbidity. Therefore, more effective interventions to prevent BCa recurrence are urgently needed.

Submucosal injection of antitumor drugs (pirarubicin) after standard TURBT was proven to be an effective approach to reducing superficial tumor recurrence (4). Gemcitabine is a pivotal chemotherapeutic agent widely used for $\mathrm{BCa}$ due to its low toxicity in general (5) and as an intravesical instillation (3). Data from our experimental and clinical studies also demonstrated that submucosal injection of gemcitabine prior to TURBT significantly reduced BCa recurrence (6). However, the underlying mechanisms are largely unknown.

Metabolomics is a newly emerging technology, which enables the identification of endogenous compounds and potentially novel mechanisms associated with disease processes (7). Metabolomics has been used to profile metabolites in various biological samples, such as serum (8), urine (9-15) and tissue $(16,17)$, which are the results of the metabolic response of living systems to drug toxicity or disease (10). Potential biomarkers identified from metabolomic profiling studies on BCa may be of diagnostic value and act as indicators of cancer recurrence (18). Currently, a number of analytical platforms, such as high-performance liquid chromatography/mass spectrometry (HPLC/MS) (10), ultra-performance LC-MS (UPLC-MS) (12), and UPLC time-of-flight MS (UPLC-TOF-MS) (15), have been employed to study the metabolomics of BCa by using urine samples. However, metabolomic studies on BCa tissues is relatively scarce $(16,17)$. Notably, different metabolomic platforms with their unique analytical approaches provide complementary insights into metabolome changes $(9,11-15)$. Therefore, there is a need to study the tissue-based metabolic signatures of $\mathrm{BCa}$ using a new metabolomics platform.

Metabolomics has also been applied to cancer treatment and drug target discovery. Eidelman et al reported using metabolomics to screen the potential therapeutic pathways in prostate cancer (PCa) (19). Metabolomics has also been proven to be a promising approach to developing reliable therapeutic targets for PCa treatment (20). The present study employed liquid chromatography (LC)-Q-Exactive MS-based metabolomic technology to study the metabolic changes in BCa tissues before and after treatment with gemcitabine. Identification of the key metabolites may reveal new metabolic changes associated with $\mathrm{BCa}$ and uncover the changes that mediate the effect of gemcitabine in the treatment of $\mathrm{BCa}$.

\section{Materials and methods}

Clinical samples. A total of 12 patients ( 9 men and 3 women; age range, 55-85 years) who had undergone TURBT at the Affiliated
Huai'an No. 1 People's Hospital of Nanjing Medical University were recruited between December 2016 and September 2017. Bladder tissue samples were collected from the same patient immediately prior to and $30 \mathrm{~min}$ after submucosal injection of gemcitabine (50 mg, dissolved in $20 \mathrm{ml}$ normal saline). The bladder tissues included $\mathrm{BCa}$ as well as adjacent non-cancerous bladder tissues. Therefore, a total of 48 samples were collected in four groups: Pre-gemcitabine normal, pre-gemcitabine $\mathrm{BCa}$, post-gemcitabine normal, and post-gemcitabine $\mathrm{BCa}$. Histopathological diagnosis was conducted by two independent pathologists according to the classification criteria of the World Health Organization/International Society of Urological Pathology (21). Written informed consent was obtained from each participant prior to recruitment. The Ethics Committee of The Affiliated Huai'an No. 1 People's Hospital of Nanjing Medical University reviewed and approved the study protocol (serial no. YL-P-2013-21-01). The sample size of the present study was similar to that of a previous study on PCa tissues, which produced valuable findings (22). In addition, the experimental design of self-control and complete collection of samples in our study may improve statistical power by avoiding confounding factors.

Tissue preparation for metabolomic analysis. Following harvesting, all tissues were snap-frozen in liquid nitrogen, and kept in a $-80^{\circ} \mathrm{C}$ freezer until further analysis. The sample preparation was conducted as described previously (23). Briefly, the tissues were fragmented, ultrasonicated for $5 \mathrm{~min}$ (power: $60 \%$, pulses: 6/4) in distilled water, and then $150 \mu \mathrm{l}$ homogenate and $450 \mu \mathrm{l}$ methanol (Merck KGaA) were mixed in a 1.5-ml Eppendorf tube for protein precipitation. The mixture was centrifuged at $16,000 \mathrm{x}$ for $15 \mathrm{~min}$ at $4^{\circ} \mathrm{C}$, and the supernatant was collected and dried in a vacuum centrifugal concentrator. The dry residue was reconstituted in ultra-pure water and used for metabolomic analysis.

Metabolomic analysis. The metabolomic analysis was conducted as previously reported (23). LC-HRMS analysis was performed on an UPLC Ultimate 3000 system (Dionex), coupled with a Q-Exactive mass spectrometer (Thermo Fisher Scientific, Inc.). The instrument operated at a 70,000 resolution with a full-scan acquisition ranging from 70 to $1,500 \mathrm{~m} / \mathrm{z}$. The chromatographic separation of metabolites associated with the metabolomic profiling used a multistep gradient containing ultra-pure water (mobile phase A) and acetonitrile (ACN; mobile phase $\mathrm{B}$ ), both acidified with $0.1 \%$ formic acid. The gradient operated at a flow rate of $0.4 \mathrm{ml} / \mathrm{min}$ over a $15-\mathrm{min}$ period. The metabolites were identified based on the accurate mass and the retention time compared with the commercial standards. The metabolite standards were purchased from Sigma-Aldrich; Merck KGaA, Damas-beta Co., Ltd., Aladdin Reagent Company and Adamas Reagent Co., Ltd.

Statistical analysis. Data collected from the mass spectrometer were processed for pattern recognition analysis (principal component analysis, PCA). Normalized MS data were exported to SIMCA-P+ software (V14.0, Umetrics AB) to perform $\mathrm{PCA}$ where grouping trends could be observed. The difference in metabolites between two groups was compared by paired t-test. According to previous reports $(24,25)$, the 
correlation between metabolite changes and cancer stage was analyzed based on the comparison between two groups using paired t-test based on the cancer stage classification. A P-value of $<0.05$ was considered as the threshold for statistically significant differences.

To further characterize the metabolic changes and the metabolic pathways involved, the differentiated metabolites were first annotated with Kyoto Encyclopedia of Genes and Genomes (KEGG, http://www.genome.jp/kegg/) and Human Metabolome Database (HMDB, http://www.hmdb.ca/) (date of access for databases: April 19, 2018). Data were then processed and analyzed using MetaboAnalyst 4.0 (http://www. metaboanalyst.ca/MetaboAnalyst/) by R software (v3.4.3, GitHub). Two modules of MetaboAnalyst were used, namely pathway analysis and enrichment analysis, which are based on the KEGG database and Small Molecule Pathway Database (SMPDB, http://smpdb.ca/), respectively (26). The metabolic network of the differential metabolites and altered metabolic pathways in KEGG general metabolic pathway was visualized by iPath 3.0 (http://pathways.embl.de/).

\section{Results}

Clinical characteristics of 12 subjects. The mean age of the subjects, including 9 men and 3 women, was 67 years (range, 55-85 years). In all 12 patients, the diagnosis of urothelial carcinoma was confirmed by histopathological examination. A total of 2 patients had $\mathrm{T}_{\mathrm{a}}, 1$ patient had $\mathrm{T}_{1}$, and 9 patients had $\mathrm{T}_{2}$ disease. Two patients were diagnosed with high-grade $\mathrm{T}_{2}$ tumors with squamous metaplasia. The clinical characteristics of the participants are shown in Table I, and are considered to be representative according to the general population of $\mathrm{BCa}$ in China $(2,27)$.

$P C A$. A total of 165 metabolites were detected. PCA was performed to process the metabolite data based on a mean center-scaling model, which is an unsupervised projection method employed to visually display the intrinsic similarities and differences in the dataset. As shown in Fig. 1, PCA (pre-gemcitabine normal vs. pre-gemcitabine BCa tissues) revealed a well-differentiated and clustered pattern in score plots, indicating the significant metabolome changes between these two groups.

Altered metabolites and cancer-associated metabolic pathways in BCa. UPLC-Q-Exactive analysis identified 34 differentially expressed metabolites annotated in the KEGG and HMDB databases in pre-gemcitabine $\mathrm{BCa}$ tissues compared with pre-gemcitabine adjacent normal tissues (Table II).

The 34 BCa-associated metabolites were then submitted to MetaboAnalyst for analysis. Table II lists all metabolites found to be altered in BCa. Table III and Fig. 2A show the metabolic pathways connected with these 34 metabolites, among which three pathways, namely glutathione, purine and thiamine metabolism, were significantly associated with $\mathrm{BCa}$. Furthermore, in order to expand our understanding of metabolic pathways related to $\mathrm{BCa}$, the module of enrichment analysis of MetaboAnalyst was used, which verified that glutathione metabolism was significantly associated with $\mathrm{BCa}$
(Table IV and Fig. 2B). The metabolic network of the differential metabolites and altered metabolic pathways in the KEGG general metabolic pathway map is shown in Fig. 2C.

Candidate targets of submucosal injection of gemcitabine. Based on targeted metabolomic analysis, we next analyzed these 34 differential metabolite changes in post-gemcitabine $\mathrm{BCa}$ tissues, and compared their levels with those in pre-gemcitabine normal tissues. A total of 32 metabolites maintained significant changes with identical trends in the comparison between pre-gemcitabine normal vs. pre-gemcitabine $\mathrm{BCa}$ tissues, indicating that the findings for differential metabolites in $\mathrm{BCa}$ are reliable. Importantly, the significant decrease in the levels of two metabolites associated with $\mathrm{BCa}$ recovered to insignificant levels following submucosal injection of gemcitabine (Table II and Fig. 3A, B and E-F). These were bilirubin and retinal, which may be the candidate targets of gemcitabine for the prevention of recurrence of urothelial $\mathrm{BCa}$. We further analyzed the changes in the two metabolites in association with cancer stage. In Ta/T1 stage disease, when comparing pre-gemcitabine normal vs. pre-gemcitabine $\mathrm{BCa}$ tissues, bilirubin was decreased significantly in the $\mathrm{BCa}$ tissues ( $\mathrm{P}=4.15 \mathrm{E}-2)$ (Table SI and Fig. 3C), while this decrease recovered to an insignificant level following submucosal injection of gemcitabine ( $\mathrm{P}=5.40 \mathrm{E}-1)$ (Table SI and Fig. 3D); however, at this stage, the decrease of retinal in the tumor was still not statistically significant $(\mathrm{P}=4.76 \mathrm{E}-1)$ (Table SI). In T2 stage disease, when comparing pre-gemcitabine normal vs. pre-gemcitabine $\mathrm{BCa}$ tissues, retinal was decreased significantly in the BCa tissues ( $\mathrm{P}=4.98 \mathrm{E}-2)$ (Table SI and Fig. 3G), while its change became statistically insignificant following submucosal injection of gemcitabine $(\mathrm{P}=3.22 \mathrm{E}-1)$ (Table SI and Fig. $3 \mathrm{H}$ ); however, at this stage, the decrease of retinal in tumor was not statistically significant $(\mathrm{P}=1.68 \mathrm{E}-1)$ (Table SI). These results indicate that bilirubin and retinal changes were correlated with cancer stage, and gemcitabine may exert its effect through metabolic pathways associated with cancer stage.

Effects of submucosal injection of gemcitabine on normal tissues. To identify the effects of submucosal injection of gemcitabine on normal bladder tissues, the metabolomes of normal tissues were compared pre- and post-gemcitabine treatment. A total of 10 metabolites were found to be significantly altered in the normal tissues following submucosal injection of gemcitabine, whereas only 2 metabolites were associated with $\mathrm{BCa}$ (Table V). Histamine was significantly decreased in $\mathrm{BCa}$, and its level was significantly increased in normal tissues after submucosal injection of gemcitabine (Fig. 3I and J). However, thiamine was significantly decreased in $\mathrm{BCa}$, and its level was significantly decreased in normal tissues after submucosal injection of gemcitabine (Fig. 3M and N). We further analyzed the changes in the two metabolite in relation to cancer stage. In $\mathrm{Ta} / \mathrm{T} 1$ stage disease, when comparing pre-gemcitabine normal vs. pre-gemcitabine $\mathrm{BCa}$ tissues, the changes in histamine $(\mathrm{P}=2.60 \mathrm{E}-1)$ and thiamine $(\mathrm{P}=8.39 \mathrm{E}-2)$ in the tumor were insignificant (Table SI). However, in T2 stage disease, when comparing pre-gemcitabine normal vs. pre-gemcitabine $\mathrm{BCa}$ tissues, the decrease in histamine $(\mathrm{P}=4.61 \mathrm{E}-3)$ (Table SI and Fig. 3K) and thiamine ( $\mathrm{P}=4.13 \mathrm{E}-4)$ (Table SI and Fig. 3O) in 
Table I. Clinical characteristics of $12 \mathrm{BCa}$ patients.

\begin{tabular}{|c|c|c|c|c|c|}
\hline \multirow[b]{2}{*}{ Number } & \multirow[b]{2}{*}{ Sex } & \multirow[b]{2}{*}{ Age (years) } & \multirow[b]{2}{*}{ Tumor type } & \multicolumn{2}{|c|}{ Histopathology } \\
\hline & & & & Stage & Grade \\
\hline 01 & Male & 55 & MIUC & $\mathrm{T} 2$ & High \\
\hline 02 & Male & 56 & MIUC & $\mathrm{T} 2$ & High \\
\hline 03 & Male & 59 & MIUC & $\mathrm{T} 2$ & High \\
\hline 04 & Female & 82 & MIUC & $\mathrm{T} 1$ & High \\
\hline 05 & Male & 67 & MIUC & $\mathrm{T} 2$ & High \\
\hline 06 & Male & 76 & MIUC & $\mathrm{T} 2 \mathrm{~b}$ & Squamous metaplasia \\
\hline 07 & Male & 76 & MIUC & $\mathrm{T} 2$ & High \\
\hline 08 & Female & 60 & MIUC & $\mathrm{T} 2$ & High \\
\hline 09 & Male & 67 & NMIUC & $\mathrm{Ta}$ & High \\
\hline 10 & Male & 58 & NMIUC & $\mathrm{Ta}$ & High \\
\hline 11 & Male & 85 & MIUC & $\mathrm{T} 2 \mathrm{a}$ & High \\
\hline 12 & Female & 61 & MIUC & $\mathrm{T} 2$ & Squamous metaplasia \\
\hline
\end{tabular}

MIUC, muscle-invasive urothelial carcinoma; NMIUC, non-muscle-invasive urothelial carcinoma. BCa, bladder cancer.

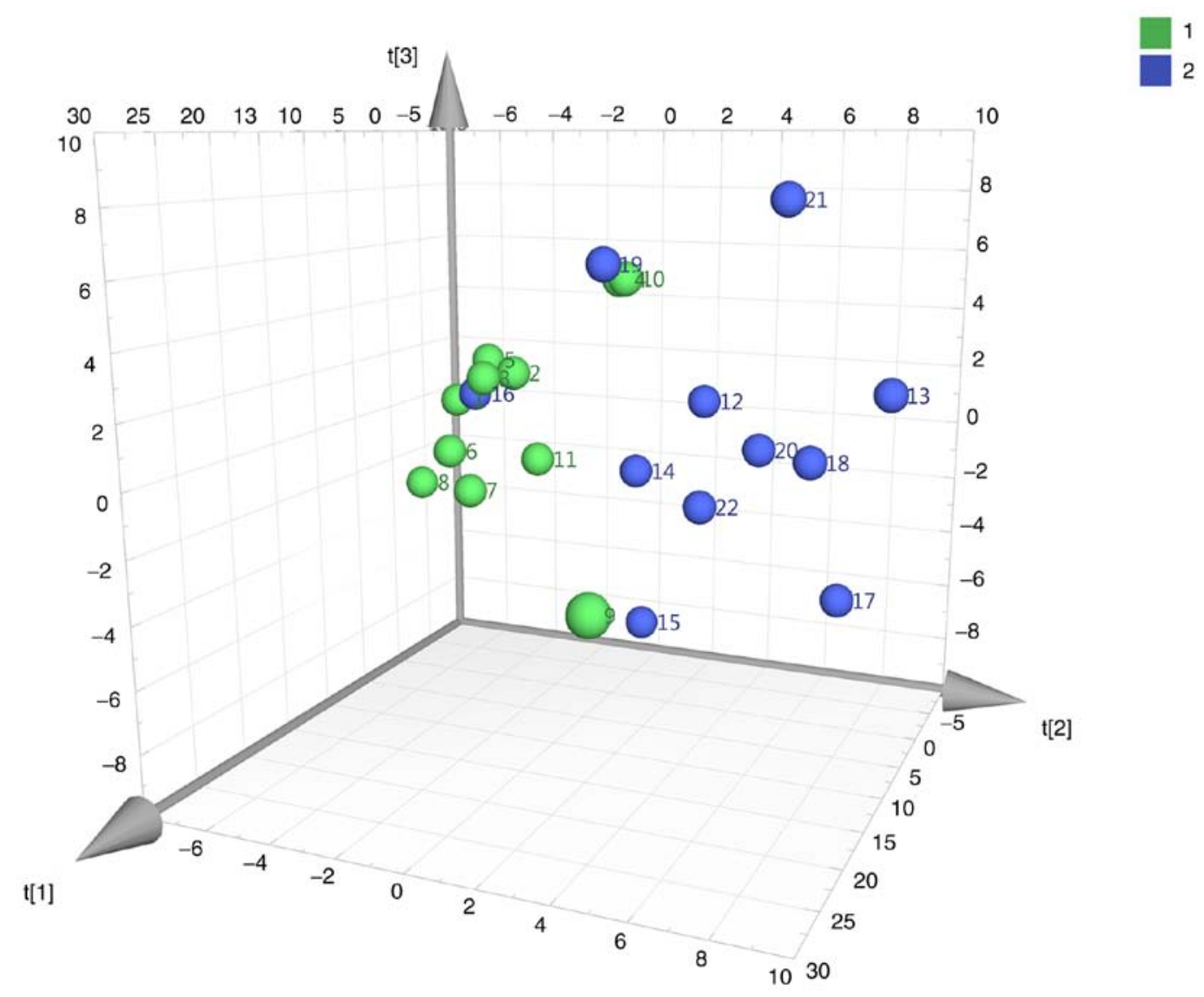

Figure 1. PCA score plots derived from pre-gemcitabine normal (green) and pre-gemcitabine BCa (blue) tissues. Comparison of pre-gemcitabine normal (green) and pre-gemcitabine $\mathrm{BCa}$ (blue) tissues. $\mathrm{PCA}$, principal component analysis; $\mathrm{BCa}$, bladder cancer.

the BCa tissues was significant. In T2 stage disease, the histamine level was significantly increased in normal tissues after submucosal injection of gemcitabine $(\mathrm{P}=3.55 \mathrm{E}-3)$ (Table SI and Fig. 3L), while thiamine was significantly decreased
$(\mathrm{P}=4.11 \mathrm{E}-2)$ (Table SI and Fig. 3P). These results indicate that these metabolite changes were correlated with cancer stage, and gemcitabine may exert its effects mainly through these metabolic pathways in $\mathrm{T} 2$ stage disease. 
Table II. List of the altered metabolites identified in BCa and their changes in the comparison between BCa tissues with or without gemcitabine pretreatment and pre-gemcitabine normal tissues.

\begin{tabular}{|c|c|c|c|c|c|c|}
\hline \multirow[b]{2}{*}{ Metabolites } & \multirow[b]{2}{*}{ KEGG } & \multirow[b]{2}{*}{ HMDB } & \multicolumn{2}{|c|}{$\begin{array}{l}\text { Pre-gemcitabine } \mathrm{BCa} \\
\text { vs. normal tissues }{ }^{\mathrm{a}}\end{array}$} & \multicolumn{2}{|c|}{$\begin{array}{l}\text { Post-gemcitabine } \mathrm{BCa} \\
\text { vs. pre-gemcitabine } \\
\text { normal tissues }^{\mathrm{a}}\end{array}$} \\
\hline & & & Fold change $^{a}$ & P-value & Fold change $^{\mathrm{a}}$ & P-value \\
\hline Deoxycytidine & C00881 & HMDB0000014 & 4.03 & $1.22 \mathrm{E}-02$ & 10.10 & $4.15 \mathrm{E}-05$ \\
\hline 5'-Methylthioadenosine & $\mathrm{C} 00170$ & HMDB0001173 & 15.16 & $2.38 \mathrm{E}-04$ & 12.22 & $4.16 \mathrm{E}-04$ \\
\hline 3'-AMP & $\mathrm{C} 01367$ & HMDB0003540 & 4.95 & $3.02 \mathrm{E}-02$ & 6.36 & 3.33E-02 \\
\hline Androstenedione & $\mathrm{C} 00280$ & HMDB0000053 & 0.16 & $4.09 \mathrm{E}-02$ & 0.10 & $5.76 \mathrm{E}-03$ \\
\hline Bilirubin & C00486 & HMDB0000054 & 0.4 & 3.97E-02 & 1.26 & 2.04E-01 \\
\hline Cholic acid & $\mathrm{C} 00695$ & HMDB0000619 & 0.29 & $3.14 \mathrm{E}-02$ & 0.24 & $2.00 \mathrm{E}-03$ \\
\hline Cytidine & $\mathrm{C} 00475$ & HMDB0000089 & 3.83 & $2.43 \mathrm{E}-03$ & 3.05 & $6.77 \mathrm{E}-03$ \\
\hline 5-Hydroxylysine & C16741 & HMDB0000450 & 0.64 & $4.40 \mathrm{E}-02$ & 0.48 & 7.49E-03 \\
\hline Deoxyinosine & $\mathrm{C} 05512$ & HMDB0000071 & 2.44 & $3.87 \mathrm{E}-02$ & 4.12 & $1.70 \mathrm{E}-02$ \\
\hline Glucosamine 6-phosphate & $\mathrm{C} 00352$ & HMDB0001254 & 6.04 & $6.26 \mathrm{E}-03$ & 6.87 & $3.02 \mathrm{E}-05$ \\
\hline Glyceraldehyde & $\mathrm{C} 02154$ & HMDB0001051 & 0.29 & $5.86 \mathrm{E}-04$ & 0.32 & $1.65 \mathrm{E}-03$ \\
\hline Sphingosine & $\mathrm{C} 00319$ & HMDB0000252 & 2.03 & $3.30 \mathrm{E}-02$ & 3.34 & $2.18 \mathrm{E}-05$ \\
\hline Glycerophosphocholine & $\mathrm{C} 00670$ & HMDB0000086 & 56.88 & $1.93 \mathrm{E}-03$ & 51.03 & $3.44 \mathrm{E}-03$ \\
\hline Glycine & $\mathrm{C} 00037$ & HMDB0000123 & 0.55 & $2.22 \mathrm{E}-02$ & 0.47 & 4.68E-03 \\
\hline Guanidine & C17349 & HMDB0001842 & 0.47 & $5.25 \mathrm{E}-03$ & 0.01 & 3.79E-04 \\
\hline Hexadecanedioic acid & C19615 & HMDB0000672 & 0.27 & $1.34 \mathrm{E}-02$ & 0.23 & $3.44 \mathrm{E}-03$ \\
\hline Histamine & $\mathrm{C} 00388$ & HMDB0000870 & 0.58 & $2.49 \mathrm{E}-02$ & 0.35 & $7.49 \mathrm{E}-03$ \\
\hline Hypotaurine & C00519 & HMDB0000965 & 0.07 & $4.19 \mathrm{E}-04$ & 0.08 & 5.33E-04 \\
\hline Inosinic acid & $\mathrm{C} 00130$ & HMDB0000175 & 92.72 & $1.73 \mathrm{E}-05$ & 150.57 & $1.43 \mathrm{E}-03$ \\
\hline L-Carnitine & $\mathrm{C} 00318$ & HMDB0000062 & 2.24 & $2.39 \mathrm{E}-03$ & 2.70 & 3.97E-03 \\
\hline L-Cystine & C00491 & HMDB0000192 & 0.49 & $2.70 \mathrm{E}-02$ & 0.13 & 1.17E-03 \\
\hline L-Phenylalanine & $\mathrm{C} 00079$ & HMDB0000159 & 0.51 & $2.80 \mathrm{E}-02$ & 0.31 & 4.70E-03 \\
\hline N-Acetylneuraminic acid & C19910 & HMDB0000230 & 2.68 & $2.13 \mathrm{E}-02$ & 2.90 & $3.38 \mathrm{E}-02$ \\
\hline Oxidized glutathione & $\mathrm{C} 00127$ & HMDB0003337 & 13.97 & $4.36 \mathrm{E}-03$ & 16.05 & 2.77E-03 \\
\hline L-Palmitoylcarnitine & $\mathrm{C} 02990$ & HMDB0000222 & 3.59 & $1.88 \mathrm{E}-02$ & 5.09 & 2.77E-04 \\
\hline Pantothenol & C05944 & HMDB0004231 & 0.44 & $3.12 \mathrm{E}-02$ & 0.18 & $8.44 \mathrm{E}-05$ \\
\hline Pyroglutamic acid & $\mathrm{C} 01879$ & HMDB0000267 & 0.29 & $1.85 \mathrm{E}-03$ & 0.16 & $5.30 \mathrm{E}-04$ \\
\hline Quinic acid & $\mathrm{C} 06746$ & HMDB0003072 & 0.09 & $4.05 \mathrm{E}-02$ & 0.25 & $4.76 \mathrm{E}-02$ \\
\hline Retinal & $\mathrm{C00376}$ & HMDB0001358 & 0.13 & 4.98E-02 & 0.43 & 3.81E-01 \\
\hline Rhamnose & $\mathrm{C} 00507$ & HMDB0000849 & 0.22 & $2.90 \mathrm{E}-03$ & 0.17 & $2.40 \mathrm{E}-04$ \\
\hline Deoxycholic acid glycine conjugate & $\mathrm{C} 05464$ & HMDB0000631 & 0.19 & $6.50 \mathrm{E}-03$ & 0.15 & $4.52 \mathrm{E}-04$ \\
\hline Sorbitol & C00794 & HMDB0000247 & 0.29 & $9.19 \mathrm{E}-03$ & 0.13 & $1.44 \mathrm{E}-03$ \\
\hline Tetradecanedioic acid & C11002 & HMDB0000872 & 0.44 & $4.46 \mathrm{E}-02$ & 0.36 & 4.47E-02 \\
\hline Thiamine & $\mathrm{C} 00378$ & HMDB0000235 & 0.34 & $6.95 \mathrm{E}-05$ & 0.18 & $2.47 \mathrm{E}-05$ \\
\hline
\end{tabular}

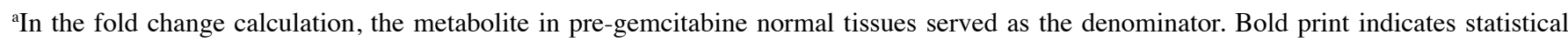
significance. BCa, bladder cancer; KEGG, Kyoto Encyclopedia of Genes and Genomes; HMDB, human metabolome database.

\section{Discussion}

In the present study, UPLC-Q-Exactive-based metabolomic analysis was utilized to profile metabolites in BCa tissues. The major findings may be summarized as follows: i) A total of 34 key metabolites associated with BCa were identified (Table II); ii) three metabolic pathways, namely glutathione, purine and thiamine metabolism, were altered in $\mathrm{BCa}$, and glutathione metabolism was the consistently altered pathway in enrichment and pathway analyses (Tables III and IV, and Fig. 2A and B); iii) among the 34 cancer-associated metabolites, the levels of bilirubin and retinal recovered after gemcitabine injection, suggesting that these two are likely the targets of gemcitabine treatment (Table II, Fig. 3A-D and E-H); iv) the effects of gemcitabine on normal bladder tissues were also investigated, and it was deduced that histamine may have the 
Table III. Pathway analysis of metabolite changes in $\mathrm{BCa}^{\mathrm{a}}$.

\begin{tabular}{|c|c|c|c|}
\hline KEGG pathway & Total & Hits & P-value \\
\hline Glutathione metabolism & 38 & 3 & $1.55 \mathrm{E}-02$ \\
\hline Purine metabolism & 92 & 4 & $3.86 \mathrm{E}-02$ \\
\hline Thiamine metabolism & 24 & 2 & $4.40 \mathrm{E}-02$ \\
\hline Nitrogen metabolism & 39 & 2 & 1.04E-01 \\
\hline Primary bile acid biosynthesis & 47 & 2 & $1.41 \mathrm{E}-01$ \\
\hline Fructose and mannose metabolism & 48 & 2 & $1.46 \mathrm{E}-01$ \\
\hline Cysteine and methionine metabolism & 56 & 2 & $1.86 \mathrm{E}-01$ \\
\hline Cyanoamino acid metabolism & 16 & 1 & 2.04E-01 \\
\hline Pyrimidine metabolism & 60 & 2 & 2.07E-01 \\
\hline Taurine and hypotaurine metabolism & 20 & 1 & $2.48 \mathrm{E}-01$ \\
\hline Retinol metabolism & 22 & 1 & $2.70 \mathrm{E}-01$ \\
\hline Ether lipid metabolism & 23 & 1 & $2.80 \mathrm{E}-01$ \\
\hline Aminoacyl-tRNA biosynthesis & 75 & 2 & $2.86 \mathrm{E}-01$ \\
\hline Alanine, aspartate and glutamate metabolism & 24 & 1 & $2.90 \mathrm{E}-01$ \\
\hline Sphingolipid metabolism & 25 & 1 & $3.01 \mathrm{E}-01$ \\
\hline Pantothenate and CoA biosynthesis & 27 & 1 & $3.20 \mathrm{E}-01$ \\
\hline Phenylalanine, tyrosine and tryptophan biosynthesis & 27 & 1 & $3.20 \mathrm{E}-01$ \\
\hline Methane metabolism & 34 & 1 & $3.86 \mathrm{E}-01$ \\
\hline Glycerophospholipid metabolism & 39 & 1 & $4.28 \mathrm{E}-01$ \\
\hline Porphyrin and chlorophyll metabolism & 104 & 2 & 4.36E-01 \\
\hline Galactose metabolism & 41 & 1 & 4.45E-01 \\
\hline Histidine metabolism & 44 & 1 & 4.68E-01 \\
\hline Phenylalanine metabolism & 45 & 1 & $4.76 \mathrm{E}-01$ \\
\hline Lysine degradation & 47 & 1 & 4.91E-01 \\
\hline Glycine, serine and threonine metabolism & 48 & 1 & 4.98E-01 \\
\hline Fatty acid metabolism & 50 & 1 & $5.13 \mathrm{E}-01$ \\
\hline Amino sugar and nucleotide sugar metabolism & 88 & 1 & 7.21E-01 \\
\hline Steroid hormone biosynthesis & 99 & 1 & 7.63E-01 \\
\hline
\end{tabular}

${ }^{a}$ The analysis was conducted by the module of pathway analysis of MetaboAnalyst 4.0. BCa, bladder cancer; KEGG, Kyoto Encyclopedia of Genes and Genomes.

ability to protect against disease recurrence, whereas thiamine may be involved in the side effects of treatment (Table $\mathrm{V}$, Fig. 3I-L and M-P), which requires further confirmation in future studies.

The identification of the three metabolic pathways significantly altered in BCa may be pathophysiologically important. Glycine is an important amino acid that participates in all three metabolic pathways. A decrease in glycine was reported as a biomarker in $\mathrm{BCa}$ (14). As a precursor of purine synthesis, reduced glycine in $\mathrm{BCa}$ indicates that a critical metabolic process associated with cell proliferation is altered in $\mathrm{BCa}$ (14). Notably, glutathione metabolism is the consistently altered pathway in enrichment and pathway analysis. Glutathione is the most abundant low-molecular-weight peptide present in eukaryotic cells (28). Glutathione is a primary cellular antioxidant that effectively scavenges free radicals and other reactive oxygen species (29) and, therefore, plays an important role in protecting cells from oxidative injury (30). Glutathione is also involved in cellular detoxification, and is required in several aspects of the immune response (31). Ke et al reported altered oxidized glutathione in $\mathrm{BCa}$; they discovered four single-nucleotide polymorphisms in the glutathione synthetase gene, and these changes were associated with $\mathrm{BCa}$ recurrence after TUR and Bacillus Calmette Guerin treatment (31). Our findings, together with others, suggest that oxidative stress in $\mathrm{BCa}$ cells is at least partially due to the disrupted glutathione metabolism.

By using LC-Q-Exactive MS, this study is, to the best of our knowledge, the first to investigate the metabolite changes in BCa treated with gemcitabine followed by TURBT. Among the 34 cancer-associated metabolites, the levels of bilirubin and retinal recovered after gemcitabine treatment, indicating that they may be the potential targets of gemcitabine for reducing $\mathrm{BCa}$ recurrence. Bilirubin, a degradation product of free heme groups, protected LLC-PK1 cells against cisplatin-induced death (32). A large population-based study demonstrated that patients with primary biliary cirrhosis (PBC) have a nine-fold increased risk of developing urinary $\mathrm{BCa}$, and $\mathrm{BCa}$ and PBC share a number of etiological factors (33). Uridine 5'-diphospho-glucuronosyltransferases (UGTs) are enzymes 

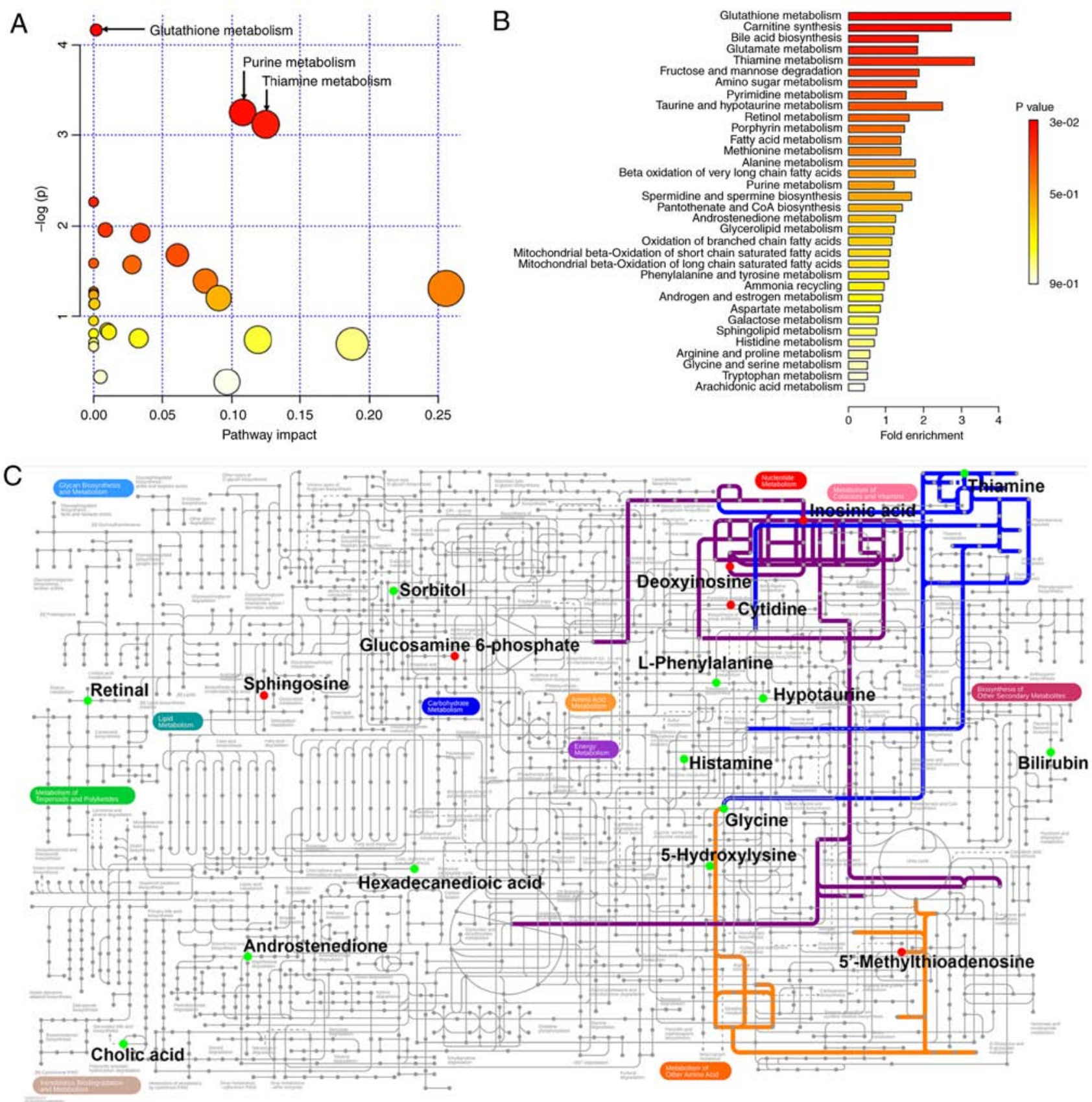

Figure 2. Results of the metabolic connection analysis of the changed metabolomic data in BCa. (A) Pathway analysis based on the KEGG database. (B) Enrichment analysis based on SMPDB. (C) Metabolic network of the differential metabolites and altered metabolic pathways in KEGG general metabolic pathway map. Red dots represent the increased metabolites in $\mathrm{BCa}$; green dots represent the specifically decreased metabolites in BCa; orange line, glutathione metabolism; purple line, purine metabolism; blue line, thiamine metabolism. The metabolites and pathways not indicated in the general pathway map are not shown. The original general metabolic pathway map is available at https://pathways.embl.de/ipath3.cgi. KEGG, Kyoto Encyclopedia of Genes and Genomes; SMPDB, Small Molecule Pathway Database; BCa, bladder cancer.

that participate in several biological processes involving bilirubin conjugation. UGTs catabolize carcinogens and, therefore, protect bladder cells from the harmful effects of toxic chemicals accumulated in the bladder. Targeting UGT1A may serve as a novel therapeutic intervention against uroepithelial carcinomas (34). Retinal (retinaldehyde; Rald) is derived from retinol (vitamin A) oxidized by alcohol dehydrogenases (35). Retinal plays an essential role in molecular signaling in vision, and serves mainly as a retinoic acid (an active form of vitamin A) precursor outside the eye (36). The serum levels of vitamin A were found to be decreased in patients with
$\mathrm{BCa}$ (37). A high dietary intake of vitamin A reduces the incidence of $\mathrm{BCa}$ (38). Ziouzenkova et al reported retinal as a distinct biological regulator involved in suppressing adipogenesis, diet-induced obesity and insulin resistance (39). The potential effect of bilirubin and retinal on the clinical outcome of patients with $\mathrm{BCa}$ identified in the present study is worthy of further investigation in the future.

The effects of gemcitabine on the metabolism of adjacent normal tissues were also examined. Most gemcitabine-induced metabolites do not overlap with those identified in $\mathrm{BCa}$, indicating that gemcitabine did not exert notable adverse effects on 
Table IV. Enrichment analysis of metabolite changes in $\mathrm{BCa}^{\mathrm{a}}$.

\begin{tabular}{|c|c|c|c|}
\hline Pathway from SMPDB & Total & Hits & P-value \\
\hline Glutathione metabolism & 21 & 3 & $2.96 \mathrm{E}-02$ \\
\hline Carnitine synthesis & 22 & 2 & 1.64E-01 \\
\hline Bile acid biosynthesis & 65 & 4 & 1.64E-01 \\
\hline Glutamate metabolism & 49 & 3 & 2.19E-01 \\
\hline Thiamine metabolism & 9 & 1 & $2.63 \mathrm{E}-01$ \\
\hline Fructose and mannose degradation & 32 & 2 & $2.88 \mathrm{E}-01$ \\
\hline Amino Sugar metabolism & 33 & 2 & $3.00 \mathrm{E}-01$ \\
\hline Pyrimidine metabolism & 59 & 3 & $3.11 \mathrm{E}-01$ \\
\hline Taurine and hypotaurine metabolism & 12 & 1 & 3.35E-01 \\
\hline Retinol metabolism & 37 & 2 & $3.50 \mathrm{E}-01$ \\
\hline Porphyrin metabolism & 40 & 2 & 3.87E-01 \\
\hline Fatty acid metabolism & 43 & 2 & 4.23E-01 \\
\hline Methionine metabolism & 43 & 2 & 4.23E-01 \\
\hline Alanine metabolism & 17 & 1 & 4.39E-01 \\
\hline Beta oxidation of very long-chain fatty acids & 17 & 1 & 4.39E-01 \\
\hline Purine metabolism & 74 & 3 & 4.51E-01 \\
\hline Spermidine and spermine biosynthesis & 18 & 1 & $4.58 \mathrm{E}-01$ \\
\hline Pantothenate and CoA biosynthesis & 21 & 1 & $5.11 \mathrm{E}-01$ \\
\hline Androstenedione metabolism & 24 & 1 & 5.59E-01 \\
\hline Glycerolipid metabolism & 25 & 1 & $5.74 \mathrm{E}-01$ \\
\hline Oxidation of branched chain fatty acids & 26 & 1 & 5.89E-01 \\
\hline Mitochondrial beta-oxidation of short-chain saturated fatty acids & 27 & 1 & $6.03 \mathrm{E}-01$ \\
\hline Mitochondrial beta-oxidation of long-chain saturated fatty acids & 28 & 1 & $6.16 \mathrm{E}-01$ \\
\hline Phenylalanine and tyrosine metabolism & 28 & 1 & $6.16 \mathrm{E}-01$ \\
\hline Ammonia recycling & 32 & 1 & $6.66 \mathrm{E}-01$ \\
\hline Androgen and estrogen metabolism & 33 & 1 & $6.78 \mathrm{E}-01$ \\
\hline Aspartate metabolism & 35 & 1 & $6.99 \mathrm{E}-01$ \\
\hline Galactose metabolism & 38 & 1 & 7.29E-01 \\
\hline Sphingolipid metabolism & 40 & 1 & 7.48E-01 \\
\hline Histidine metabolism & 43 & 1 & 7.73E-01 \\
\hline Arginine and proline metabolism & 53 & 1 & $8.41 \mathrm{E}-01$ \\
\hline Glycine and serine metabolism & 59 & 1 & $8.72 \mathrm{E}-01$ \\
\hline Tryptophan metabolism & 60 & 1 & $8.76 \mathrm{E}-01$ \\
\hline Arachidonic acid metabolism & 69 & 1 & $9.10 \mathrm{E}-01$ \\
\hline
\end{tabular}

${ }^{a}$ The analysis was conducted by the module of enrichment analysis of MetaboAnalyst 4.0. BCa, bladder cancer. SMPDB, Small Molecule Pathway Database.

normal bladder tissues. It was observed that histamine change may be associated with the prevention of relapse. Histamine is derived from the decarboxylation of histidine by histidine decarboxylase in mammals (8). Histamine is primarily released in inflammatory processes by mast cells (8), which are closely associated with $\mathrm{BCa}$ (40). Histamine $\mathrm{H} 1$ receptor (HRH1) expression was identified in $\mathrm{BCa}$ and found to be associated with the prognosis (41). It was observed that thiamine change may be involved in treatment-related side effects. Thiamine, or vitamin B-1, is a water-soluble vitamin (42). An early report by Pamukcu et al demonstrated that the incidence of urinary bladder carcinomas in rats fed bracken fern and additionally s.c. injected once weekly with $2 \mathrm{mg}$ of thiamine hydrochloride was significantly higher compared with that in rats fed bracken fern but receiving no thiamine supplements, as thiamine may interfere with the absorption, distribution, metabolism, or excretion of the bracken fern (43). However, a case-control study from New Hampshire investigated the effect of minerals and vitamins on the risk of $\mathrm{BCa}$, and found that a higher total intake of thiamine was inversely correlated with BCa risk in older participants (44).

There is currently a lack of effective biomarkers for $\mathrm{BCa}$ diagnosis and prognosis. Metabolomic profiles from tissue have the potential to be used, along with other current diagnostics, to help guide the clinical management of patients with BCa. The changed metabolites identified in the present 
Table V. Changes in normal tissues after gemcitabine treatment.

Pre-gemcitabine

BCa vs. normal tissues ${ }^{\mathrm{a}}$
Post-gemcitabine vs.

pre-gemcitabine normal tissues ${ }^{\mathrm{a}}$

Metabolites

Fold-change $^{\mathrm{a}}$

P-value

Fold-change

P-value

3-Methyladenine

1.54

3.78E-01

0.17

2.33E-02

Ascorbic acid

22.26

$1.85 \mathrm{E}-01$

0.28

$5.46 \mathrm{E}-03$

Creatinine

0.48

3.44E-01

0.13

$1.16 \mathrm{E}-02$

D-Glyceraldehyde 3-phosphate

3.33E-01

0.15

$2.16 \mathrm{E}-02$

Histamine

0.58

2.49E-02

2.26

3.73E-04

N-Acetylglutamic acid

8.58E-02

0.57

4.95E-02

0.68

$5.45 \mathrm{E}-01$

0.11

4.25E-02

0.34

6.95E-05

0.65

2.11E-02

Thiamine

0.10

7.66E-02

0.11

3.23E-02

1.41

6.74E-02

3.65E-02

${ }^{\text {an }}$ the fold change calculation, the metabolite in pre-gemcitabine normal tissues served as the denominator. Bold print indicates statistically significant differences. $\mathrm{BCa}$, bladder cancer.
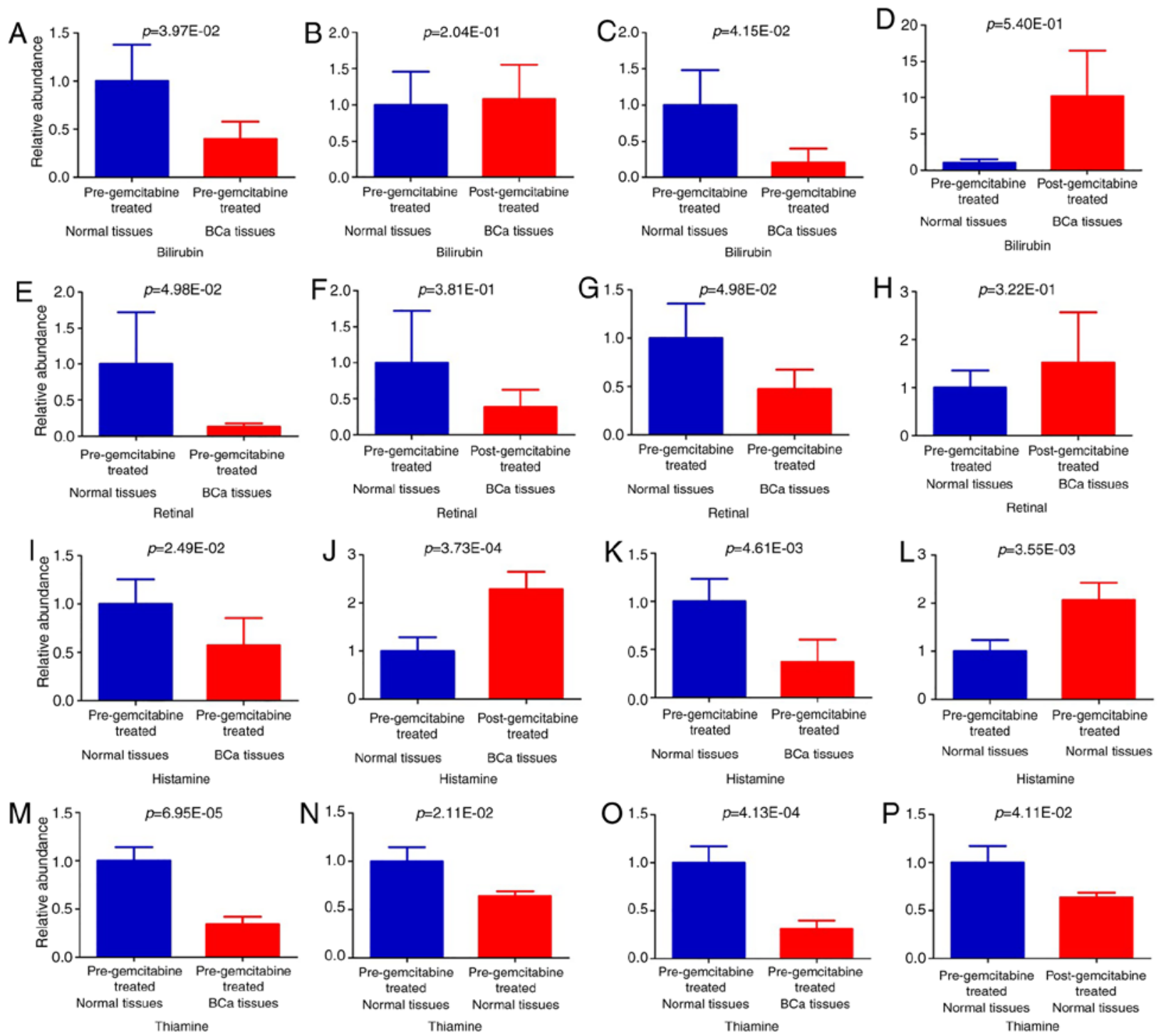

Figure 3. Relative levels of potential candidate targets of submucosal injection of gemcitabine (A, B, E, F, I, J, M and N). All samples; (C and D) Ta/T1 stage; $(\mathrm{G}, \mathrm{H}, \mathrm{K}, \mathrm{L}, \mathrm{O}$ and $\mathrm{P}) \mathrm{T} 2$ stage. Values are presented as mean \pm standard error of the mean. The P-values of paired t-test are indicated. 
study, such as glycine, may be used as potential biomarkers for $\mathrm{BCa}$. In addition, those metabolites discovered in $\mathrm{BCa}$ with gemcitabine treatment may reveal new metabolic pathways that mediate the anti-recurrence effect of gemcitabine, which currently remain elusive.

In summary, the present study employed UPLC-HRMSbased metabolomic analysis to investigate metabolite changes in bladder tissues from $\mathrm{BCa}$ and $\mathrm{BCa}$ treated with gemcitabine. The findings may provide new insights into metabolic changes in $\mathrm{BCa}$ and the biomolecular basis of submucosal injection of gemcitabine for $\mathrm{BCa}$. In addition, the study demonstrated that the UPLC-HRMS-based metabolomic analysis provides comprehensive metabolite profiling data that may pave the way to a novel approach to $\mathrm{BCa}$ research.

\section{Acknowledgements}

Not applicable.

\section{Funding}

The present study was supported by grants from the National Natural Science Foundation (81872650 and 81573182); the Natural Science Foundation of the Jiangsu Higher Education Institutions of China (18KJA320003 and 18KJB320001); the Key Research \& Development Plan of Jiangsu Province (BE2017628); the Southeast University \& Nanjing Medical University Collaborative Research Project (2242018K3DN25); the Priority Academic Program Development of Jiangsu Higher Education Institutions (PAPD); the 333 Project of Jiangsu Province (BRA2017241); the Natural Science Foundation of Huai'an (HAB201801); the Huai'an Promotion Project for Science and Technology International Cooperation (HAC201708); and the Innovation Fund Project of the State Key Laboratory of Reproductive Medicine (SKLRM-GC201901).

\section{Availability of data and materials}

The datasets generated and/or analyzed during the present study are available from the corresponding author on reasonable request.

\section{Authors' contributions}

CY, XS, KW, YG, RS and MC designed and performed the experiments. TL and MC analyzed and interpreted the raw data. SZ, XJ, BZ and HW collected the bladder tissue samples. $\mathrm{ZG}$ and SS performed the histopathological examination of BCa tissues. JL and JT preserved the samples and collected the basic information of the participants. CY, TL and MC wrote the manuscript. XW, HJ, XN, XW, MC and GF critically revised the manuscript for important intellectual content. MC and GF supervised the project. All authors have read and approved the final version of the manuscript for publication.

\section{Ethics approval and consent to participate}

The Ethics Committee of The Affiliated Huai'an No. 1 People's Hospital of Nanjing Medical University reviewed and approved the study protocol (serial no. YL-P-2013-21-01).

\section{Patient consent for publication}

Not applicable.

\section{Competing interests}

The authors declare that they have no competing interests.

\section{Authors' information}

Chao Yang: 15949177271@163.com; Xian Sun: 1019949991@ qq.com; Hengbing Wang: wanghengbing2004@163.com; Ting Lu: 552095014@qq.com; Keqing Wu: wkwly1993@ qq.com; Yusheng Guan: guanys@njmu.edu.cn; Jing Tang: tjing19681222@sina.com; Jian Liang: 1j3936@126.com; Rongli Sun: 101012172@seu.edu.cn; Zhongying Guo: guozhongying407@163.com; Sinian Zheng: zhengsinian@163. com; Xiaoli Wu: wuxiaoli2233@126.com; Hesong Jiang: njjhs2007@163.com; Xi Jiang: piscesjxi@163.com; Bing Zhong: 15152569186@163.com; Xiaobing Niu: bingke2008@ sina.com; Suan Sun: hayyssa@163.com; Xinru Wang: xrwang@njmu.edu.cn

\section{References}

1. Ferlay J, Soerjomataram I, Dikshit R, Eser S, Mathers C, Rebelo M, Parkin DM, Forman D and Bray F: Cancer incidence and mortality worldwide: Sources, methods and major patterns in GLOBOCAN 2012. Int J Cancer 136: E359-E386, 2015.

2. Messing EM, Madeb R, Young T, Gilchrist KW, Bram L, Greenberg EB, Wegenke JD, Stephenson L, Gee J and Feng C: Long-term outcome of hematuria home screening for bladder cancer in men. Cancer 107: 2173-2179, 2006.

3. Messing EM, Tangen CM, Lerner SP, Sahasrabudhe DM, Koppie TM, Wood DP Jr, Mack PC, Svatek RS, Evans CP, Hafez KS, et al: Effect of intravesical instillation of gemcitabine vs saline immediately following resection of suspected low-grade non-muscle-invasive bladder cancer on tumor recurrence: SWOG S0337 randomized clinical trial. JAMA 319: 1880-1888, 2018.

4. Chen X, Wang B, Tian HZ and Gao JZ: Submucosal injection of anti-tumor drug on the prevention of Post-TUR-Bt recurrence. Zhonghua Wai Ke Za Zhi 42: 580-582, 2004 (In Chinese).

5. Caffo O, Thompson C, De Santis M, Kragelj B, Hamstra DA, Azria D, Fellin G, Pappagallo GL, Galligioni E and Choudhury A: Concurrent gemcitabine and radiotherapy for the treatment of muscle-invasive bladder cancer: A pooled individual data analysis of eight phase I-II trials. Radiother Oncol 121: 193-198, 2016.

6. Sternberg CN, Bellmunt J, Sonpavde G, Siefker-Radtke AO, Stadler WM, Bajorin DF, Dreicer R, George DJ, Milowsky MI, Theodorescu D, et al: ICUD-EAU international consultation on bladder cancer 2012: Chemotherapy for urothelial carcinoma-neoadjuvant and adjuvant settings. Eur Urol 63: 58-66, 2013.

7. Van QN and Veenstra TD: How close is the bench to the bedside? Metabolic profiling in cancer research. Genome Med 1: 5, 2009.

8. Bansal N, Gupta A, Mitash N, Shakya PS, Mandhani A, Mahdi AA, Sankhwar SN and Mandal SK: Low- and high-grade bladder cancer determination via human serum-based metabolomics approach. J Proteome Res 12: 5839-5850, 2013

9. Jin X, Yun SJ, Jeong P, Kim IY, Kim WJ and Park S: Diagnosis of bladder cancer and prediction of survival by urinary metabolomics. Oncotarget 5: 1635-1645, 2014.

10. Issaq HJ, Nativ O, Waybright T, Luke B, Veenstra TD, Issaq EJ, Kravstov A and Mullerad M: Detection of bladder cancer in human urine by metabolomic profiling using high performance liquid chromatography/mass spectrometry. J Urol 179: 2422-2426, 2008. 
11. Pasikanti KK, Esuvaranathan K, Hong Y, Ho PC, Mahendran R, Raman Nee Mani L, Chiong E and Chan EC: Urinary metabotyping of bladder cancer using two-dimensional gas chromatography time-of-flight mass spectrometry. J Proteome Res 12: 3865-3873, 2013.

12. Liu X, Cheng X, Liu X, He L, Zhang W, Wang Y, Sun W and Ji Z Investigation of the urinary metabolic variations and the application in bladder cancer biomarker discovery. Int J Cancer 143 408-418, 2018

13. Alberice JV, Amaral AF, Armitage EG, Lorente JA, Algaba F Carrilho E, Márquez M, García A, Malats $\mathrm{N}$ and Barbas C: Searching for urine biomarkers of bladder cancer recurrence using a liquid chromatography-mass spectrometry and capillary electrophoresis-mass spectrometry metabolomics approach J Chromatogr A 1318: 163-170, 2013.

14. Zhou Y, Song R, Ma C, Zhou L, Liu X, Yin P, Zhang Z, Sun Y, $\mathrm{Xu} C, \mathrm{Lu} X$ and Xu G: Discovery and validation of potential urinary biomarkers for bladder cancer diagnosis using a pseudotargeted GC-MS metabolomics method. Oncotarget 8 20719-20728, 2017.

15. Shao CH, Chen CL, Lin JY, Chen CJ, Fu SH, Chen YT, Chang YS, Yu JS, Tsui KH, Juo CG and Wu KP: Metabolite marker discovery for the detection of bladder cancer by comparative metabolomics. Oncotarget 8: 38802-38810, 2017.

16. von Rundstedt FC, Rajapakshe K, Ma J, Arnold JM, Gohlke J, Putluri V, Krishnapuram R, Piyarathna DB, Lotan Y, Gödde $\mathrm{D}$, et al: Integrative pathway analysis of metabolic signature in bladder cancer: A linkage to the cancer genome atlas project and prediction of survival. J Urol 195: 1911-1919, 2016.

17. Putluri N, Shojaie A, Vasu VT, Vareed SK, Nalluri S, Putluri V, Thangjam GS, Panzitt K, Tallman CT, Butler C, et al: Metabolomic profiling reveals potential markers and bioprocesses altered in bladder cancer progression. Cancer Res 71: 7376-7386, 2011

18. Cheng Y, Yang X, Deng X, Zhang X, Li P, Tao J, Qin C, Wei J and Lu Q: Metabolomics in bladder cancer: A systematic review. Int J Clin Exp Med 8: 11052-11063, 2015.

19. Eidelman E, Tripathi H, Fu DX and Siddiqui MM: Linking cellular metabolism and metabolomics to risk-stratification of prostate cancer clinical aggressiveness and potential therapeutic pathways. Transl Androl Urol 7 (Suppl 4): S490-S497, 2018.

20. Lima AR, Bastos Mde L, Carvalho $M$ and Guedes de Pinho P: Biomarker discovery in human prostate cancer: An update in metabolomics studies. Transl Oncol 9: 357-370, 2016.

21. Epstein JI, Amin MB, Reuter VR and Mostofi FK: The world health organization/international society of urological pathology consensus classification of urothelial (transitional cell) neoplasms of the urinary bladder. Bladder consensus conference committee. Am J Surg Pathol 22: 1435-1448, 1998.

22. Sreekumar A, Poisson LM, Rajendiran TM, Khan AP, Cao Q, Yu J, Laxman B, Mehra R, Lonigro RJ, Li Y, et al: Metabolomic profiles delineate potential role for sarcosine in prostate cancer progression. Nature 457: 910-914, 2009.

23. Hu W, Dong T, Wang L, Guan Q, Song L, Chen D, Zhou Z, Chen M, Xia Y and Wang X: Obesity aggravates toxic effect of BPA on spermatogenesis. Environ Int 105: 56-65, 2017.

24. Christen S, Lorendeau D, Schmieder R, Broekaert D, Metzger K, Veys K, Elia I, Buescher JM, Orth MF, Davidson SM, et al: Breast cancer-derived lung metastases show increased pyruvate carboxylase-dependent anaplerosis. Cell Rep 17: 837-848, 2016.

25. Davidson SM, Papagiannakopoulos T, Olenchock BA, Heyman JE, Keibler MA, Luengo A, Bauer MR, Jha AK, O'Brien JP, Pierce KA, et al: Environment impacts the metabolic dependencies of Ras-driven non-small cell lung cancer. Cell Metab 23: 517-528, 2016.

26. Frolkis A, Knox C, Lim E, Jewison T, Law V, Hau DD, Liu P, Gautam B, Ly S, Guo AC, et al: SMPDB: The small molecule pathway database. Nucleic Acids Res 38 (Database Issue): D480-D487, 2010.
27. He YT, Li DJ, Liang D, Zheng RS, Zhang SW, Zeng HM, Chen WQ and He J: Incidence and mortality of bladder cancer in China, 2014. Zhonghua Zhong Liu Za Zhi 40: 647-652, 2018 (In Chinese)

28. Estrela JM, Ortega A and Obrador E: Glutathione in cancer biology and therapy. Crit Rev Clin Lab Sci 43: 143-181, 2006.

29. Balendiran GK, Dabur R and Fraser D: The role of glutathione in cancer. Cell Biochem Funct 22: 343-352, 2004

30. Amelio I, Cutruzzolá F, Antonov A, Agostini M and Melino G: Serine and glycine metabolism in cancer. Trends Biochem Sci 39: 191-198, 2014.

31. Ke HL, Lin J, Ye Y, Wu WJ, Lin HH, Wei H, Huang M, Chang DW, Dinney CP and Wu X: Genetic variations in glutathione pathway genes predict cancer recurrence in patients treated with transurethral resection and bacillus calmette-guerin instillation for non-muscle invasive bladder cancer. Ann Surg Oncol 22: 4104-4110, 2015.

32. Patricia Moreno-Londoño A, Bello-Alvarez $\mathrm{C}$ and PedrazaChaverri J: Isoliquiritigenin pretreatment attenuates cisplatin induced proximal tubular cells (LLC-PK1) death and enhances the toxicity induced by this drug in bladder cancer T24 cell line. Food Chem Toxicol 109: 143-154, 2017.

33. Boonstra K, Bokelaar R, Stadhouders PH, Tuynman HA, Poen AC, van Nieuwkerk KM, Witteman EM, Hamann D, Witteman BJ, Beuers U and Ponsioen CY: Increased cancer risk in a large population-based cohort of patients with primary biliary cirrhosis: Follow-up for up to 36 years. Hepatol Int 8: 266-274, 2014

34. Sundararaghavan VL, Sindhwani $\mathrm{P}$ and Hinds TD Jr: Glucuronidation and UGT isozymes in bladder: New targets for the treatment of uroepithelial carcinomas? Oncotarget 8: 3640-3648, 2017

35. Duester G, Mic FA and Molotkov A: Cytosolic retinoid dehydrogenases govern ubiquitous metabolism of retinol to retinaldehyde followed by tissue-specific metabolism to retinoic acid. Chem Biol Interact 143-144: 201-210, 2003.

36. Napoli JL: Retinoic acid: Its biosynthesis and metabolism. Prog Nucleic Acid Res Mol Biol 63: 139-188, 1999.

37. Mahmoud LA and Robinson WA: Vitamin A levels in human bladder cancer. Int J Cancer 30: 143-145, 1982.

38. Mettlin C and Graham S: Dietary risk factors in human bladder cancer. Am J Epidemiol 110: 255-263, 1979.

39. Ziouzenkova O, Orasanu G, Sharlach M, Akiyama TE, Berger JP Viereck J, Hamilton JA, Tang G, Dolnikowski GG, Vogel S, et al: Retinaldehyde represses adipogenesis and diet-induced obesity. Nat Med 13: 695-702, 2007.

40. Serel TA, Soyupek S and Çandir Ö: Association between mast cells and bladder carcinoma. Urol Int 72: 299-302, 2004.

41. Wang M, Wei X, Shi L, Chen B, Zhao G and Yang H: Integrative genomic analyses of the histamine $\mathrm{H} 1$ receptor and its role in cancer prediction. Int J Mol Med 33: 1019-1026, 2014.

42. Kerns JC and Gutierrez JL: Thiamin. Adv Nutr 8: 395-397, 2017.

43. Pamukcu AM, Yalciner S, Price JM and Bryan GT: Effects of the coadministration of thiamine on the incidence of urinary bladder carcinomas in rats fed bracken fern. Cancer Res 30: 2671-2674, 1970.

44. Brinkman MT, Karagas MR, Zens MS, Schned A, Reulen RC and Zeegers MP: Minerals and vitamins and the risk of bladder cancer: Results from the new hampshire study. Cancer Causes Control 21: 609-619, 2010.

This work is licensed under a Creative Commons Attribution-NonCommercial-NoDerivatives 4.0 International (CC BY-NC-ND 4.0) License. 